Table S1 Closest relative sequences obtained with NCBI-Blast search using generated partial 18S rRNA sequences from water samples of Arraial do Cabo region. PO: region influenced by port activity and sewage disposal. RE: open ocean region. S: surface. F: bottom
Phylogenetic
Closest described relative
Closest phylotype

Species or strain (accession no.)

Sim

Sequence (accession no.)

Sim

POS

POF RES

REF

\section{Metazoa/Fungi group}

Incertae sedis

Metazoa

Annelida

Brachiopoda

Cnidaria
Amoebidium parasiticum Y19155 Ichthyophonida sp. EU124916

Ichthyophonus hoferi EU332789

Pseudoperkinsus tapetis AF192386

Anaitides sp. AY894293

Chrysoxys sp. AF123302

Dasybranchus caducus AF448153

Hydroides norvegica AY611452

Karlodinium micrum EF492490

Karlodinium micrum EF492490

Lecudina polymorpha AY196707

Pectinaria gouldii DQ790091

Pholoe baltica AY176301

Pomatoceros lamarckii DQ140404

Selenidium serpulae DQ683562

Spiophanes kroeyeri EU340096

Clausocalanus ingens AF367718

Ctenocalanus vanus AF462320

Cytheromorpha acupunctata AB076630

Ichthybotus hudsoni AY749892

Neocalanus cristatus AF514344

Paracyclopina nana FJ214952

Phthirus pubis AF139485

Hemithiris psittaceae U08322

Clytia hummelincki AY789745

Cordagalma cordiforme AY937317

Geryonia proboscidalisvEU247816

Junceella aquamata AY962535

Liriope tetraphylla AY920756

\section{1}

90-95

$88-91$

86-89

94

97

98

92

98

83

88

96

99

98

93

98

97-99

91-99

93-96

96

99

76

88

99

99

99
Unc. Eukaryote DQ234281

Unc. Eukaryote AY884988

Unc. Eukaryote AY884988 / AY331730

Unc. Eukaryote DQ234281 / FJ221394

Anaitides sp. AY894293

Unc. Stramenopile EU143916

Dasybranchus caducus AF448153

Hydroides norvegica AY611452

Karlodinium micrum EF492490

Unc. Eukaryote AJ402340

Unc. Eukaryote EU050982

Pectinaria gouldii DQ790091

Pholoe baltica AY176301

Pomatoceros lamarckii DQ140404

Selenidium serpulae DQ683562

Spiophanes kroeyeri EU340096

Clausocalanus ingens AF367718

Ctenocalanus vanus AF462320

Cytheromorpha acupunctata AB076630

Ichthybotus hudsoni AY749892

Unc. Eukaryote AY665127 / AY665127

Unc. Ekaryote DQ344714

Unc. Pucciniomycotina EU647044

Hemithiris psittaceae U08322

Unc. Eukaryote EU189028

Unc. Eukaryote AY665131

Geryonia proboscidalisvEU247816

Junceella aquamata AY962535

Liriope tetraphylla AY920756
No. of clones

$\begin{array}{ccccc}94 & 2 & & & \\ 93-99 & 103 & 35 & 1 & 2 \\ 96-98 & 104 & 32 & 3 & - \\ 95-96 & - & - & 14 & - \\ 94 & & & & 2\end{array}$


Chordata

Mollusca

Choanoflagellida Fungi

Ascomycota

Basidiomycota

Chytridiomycota

Incertae sedis

Unclass. Fungi

Haptophyceae

Prymnesiales

Viridiplantae

Chlorophyta

Streptophyta
Human DNA sequence CT476837

Hiatella arctica AM77451

Ostrea edulis U88709

Perna canaliculus DQ640523

Pyrunculus sp. DQ923465

Stephanoeca diplocostata AF084235

Dothioraceae sp. EF060795

Pneumocystis carinii X12708

Pleosporales sp. EU594362

Taphrina johansonii AJ495835

Cryptococcus vishniacii EU723509

Sporisorium sp FJ517760

Ustilago maydis X62396

Ustilago sp. AJ 244777

Chytriomyces hyalinus DQ536487

Basidiobolus haptosporus AF368504

Fungal sp. GQ120167

Fungal sp. GQ120167

Chrysochromulina sp DQ980478

Chlamydomonas sp. AF514399

Chlorella sp. AB437244

Coccomyxa parasitica EU127469

Mantoniella squamata X73999

Nannochloris sp. AB183585

Ostreococcus lucimarinus CP000592

Ostreococcus lucimarinus CP000592

Picochlorum sp. AB488603

Prasinophyta sp. AY425302

Pycnococcus provasolii AY425305

Pycnococcus sp. AB058359

Pyramimonas aureus AB052289

Tetraselmis sp. AY425299

Luffa quinquefida AF008957

Mesotaenium chlamydosporum AJ553923

Netrium interruptum AJ428071

Potamogeton zosteriformis EF526358

Staurastrum pingue AJ428109
96-99 Human DNA sequence CT476837

Hiatella arctica AM774511

Ostrea edulis 18S U88709

Perna canaliculus DQ640523

Pyrunculus sp. DQ923465

Unc. Eukaryote EF526904

Unc. Ascomycete EU409872

Pneumocystis carinii X12708

Pleosporales sp. EU594362

Taphrina johansonii AJ495835

Unc. Agaricomycotina EU647139

Unc. Eukaryote EU189028

Ustilago maydis X62396

Ustilago sp. AJ244777

Unc. Eukaryote EU091849

Unc. E

Fungal sp. GQ120167

Unc. Eukaryote EU189028

Chrysochromulina sp DQ980478

Unc. Eukaryote AB238085

Chlorella sp. AB437244

Coccomyxa parasitica EU127469

Mantoniella squamata X73999

Unc. Eukaryote FJ221502 / AB275076

Ostreococcus lucimarinus CP000592

Unc. Eukaryote AF525861

Unc. Eukaryote AB275076

Unc. Prasinophyte AY425303

Pycnococcus provasolii AY425305

Pycnococcus sp. AB058359

Pyramimonas aureus AB052289

Tetraselmis sp. AY425299

Unc. Eukaryote FJ785978

Unc. Eukaryote AY665068

Environmental Phaseoleae EF024614

Unc. Cercozoan EU567235

Unc. Eukaryote AJ829837

$\begin{array}{ccccc}96-99 & 2 & 21 & 2 & 3 \\ 99 & & 9 & & \\ 99 & 1 & & \\ 100 & & & 4 & 1 \\ 97 & & & & \\ 90 & & 1 & & \\ & & & & 1 \\ 98 & & & & 1 \\ 91 & & 2 & & \\ 93 & & & 2 & \\ 88 & & & & 1 \\ 100 & & & & 1 \\ 96 & 8 & 6 & 3 & 9 \\ 97 & 2 & & & \\ 96 & & & 3 & \\ 88 & & & 4 & 106 \\ 98 & 39 & 117 & 83 & \end{array}$

99

96

95
3 
Alveolata

Apicomplexa

Ciliophora

Dinophyceae

Stramenopiles

Bacillariophyta
Colpodella edax AY234843

Cryptosporidium serpentis AF093501

Ophriocystis elektroscirrha AF129883

Parastrombidinopsis minima DQ393786

Parastrombidinopsis minima DQ393786

Tintinnopsis tubulosoides AF399111

Varistrombidium sp. DQ811090

Amoebophrya sp. AF472554

Amoebophrya sp."

Cachonina sp. AB183639 / AF033865

Ceratium furca AJ276699

Duboscquella sp. AB295041

Gymnodinium aureolum DQ779991

Gymnodinium aureolum DQ779991

Gymnodinium catenatum DQ779990

Gymnodinium sp. EF492493

Gymnodinium sp. AF274260

Gyrodinium helveticum AB120004

Gyrodinium fusiforme AB120002

Gyrodinium galatheanum AF274262

Heterocapsa niei EF492499

Heterocapsa rotundata AF274267

Karlodinium micrum EF492490

Karlodinium micrum EF492506

Lessardia elongata AF521100

Peridinium quinquecorne AB246746

Polarella glacialis AY179607 / AF099183

Prorocentrum mexicanum Y16232

Prorocentrum triestinum EF492512

Pyrophacus steinii AY44302

Roscoffia capitata AF521101

Warnowia sp. FJ947040

Attheya septentrionalis AY485517

Fragilariopsis cylindrus EF140624

Minutocellus sp. AY485520

Pseudo-nitzschia pungens U18240

Skeletonema japonicum DQ396518
Unc. Eukaryote AB252763

Unc. Eukaryote AY331776

Unc. Eukaryote EF100310

Parastrombidinopsis mínima DQ393786

Unc. Eukaryote FJ765410 / AJ829840

Unc. Eukaryote FJ939124

Unc. Eukaryote AY129053

Amoebophrya sp. AF472554

Unc. Eukaryote

82-88 Unc. Alveolate DQ186536

$\begin{array}{cl}82-88 & \text { Unc. Alveolate DQ186536 } \\ 99 & \text { Unc. Eukaryote EF526910 }\end{array}$

86-93 Unc. Eukaryote EF527151 / AY665057 / EU33303

Gymnodinium aureolum DQ779991

Unc. Eukaryote AY665030

Unc. Eukaryote FJ785963

Gymnodinium sp. EF492493

Unc. Eukaryote AY331745

Unc. Eukaryote AY664972

96

96-100

Unc. Eukaryote EF527094 / EF527101

Unc. Eukaryote AY664962

Unc. Eukaryote EF527111

Heterocapsa rotundata AF274267

Karlodinium micrum EF492490

Unc. Eukaryote AY789781

Unc. Eukaryote ${ }^{* 2}$

Unc. Eukaryote FJ221800

Unc. Eukaryote DQ310254 / AJ402340

Prorocentrum mexicanum Y16232

Unc. Eukaryote EF527089

Pyrophacus steinii AY443024

Unc. Eukaryote AY665030 / AB275022 / EU785270

Unc. Eukaryote AY664877

Unc. Eukaryote DQ344759

$99 \quad$ Fragilariopsis cylindrus EF140624

99 $\quad$ Fragilariopsis cylindrus EF140624

$98 \quad$ Unc. Eukaryote EU182819

$98 \quad$ Skeletonema japonicum DQ396518

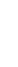

1

$\begin{array}{ll}5 & 8 \\ 1 & 1\end{array}$


Chrysophyceae

Oomycetes

Labyrinthulida

Pelagophyceae

Unclassified

\section{Rhizaria}

Acantharea

Cercozoa

Gromiidae

Foraminifera

Polycystinea

Unclass. Rhizaria

Cryptophyta

Unclass. Eukaryotes

Cryomonadida
Paraphysomonas imperforata EF432519

Achlya bisexualis M32705

Haliphthoros sp. AB178865

Aplanochytrium kerguelense $\mathrm{AB} 022103$

Aplanochytrium stocchinoi AJ519935

Labyrinthuloides minuta L27634

Labyrinthuloides yorkensis AF265333

Oblongichytrium sp. AB290575

Schizochytrium aggregatum AF265336

Thraustochytriidae sp. DQ367047

Hyphochytrium catenoides AF163294

Rhizidiomyces apophysatus AF163295

Coccoid pelagophyte U40927

Pelagomonas calceolata EF455763

Pirsonia diadema AJ561114

Chaunacanthid sp. AF018158

Allas sp. AY268040

Phagomyxa bellerocheae AF310903

Cercomonas sp. AF411266

Cercozoa sp. EU709238

Protaspis obliqua FJ824122

Protaspis sp. FJ824125

Gromia oviformis AJ457811 / AJ457812

Ammonia beccarii U07937

Dictyocoryne profunda AB101540

Paradinium poucheti EU189031

Teleaulax amphioxeia AJ421146
Lagenidium myophilum AB284577
Unc. Eukaryote AY789782

Achlya bisexualis M32705

Haliphthoros sp. AB178865

Lagenidium myophilum AB284577

Unc. Eukaryote AY129068

Unc. Labyrinthulid FJ800649 / FJ800598

Unc. Eukaryote AB191425

Unc. Eukaryote DQ103777 / AY129068

Unc. Eukaryote AY665006

Unc. Eukaryote DQ344759

Unc. Eukaryote EF526909 / 504337

Unc. Eukaryote

Unc. Eukaryote EF526909 / EU162648

Pelagomonas calceolata EF455763

Unc. Eukaryote AY331765

Symphyacanthid AF063242.1

Unc. Eukaryote FJ222163

Phagomyxa bellerocheae AF310903

Unc. Cercozoan EU785280

Unc. Cercozoan AY620336

Unc. Eukaryote FJ985885 / AY620309

Unc. Eukaryote AY885064 / DQ234284

Gromia oviformis AJ457811 / AJ457812

Unc. Eukaryote AY179972 / EF100299

Um. Eukaryote EU333071

Unc. Eukaryote EU798716

Unc. eukaryote DQ310323

Unc. Eukaryote AY885052
Coccoid pelagophyte U40927

85
93
$93-96$
97
969
90
$92-95$
96
96
93
93
89
92
93
93
93
$92-98$
$92-97$
990

AF0695161, AF472554;

*2 - FJ785975, AY129041, EF172843, EF172848, EU793779, AY665001, EU793261, EU785256, EU793852, EU333103, EF172945, AY129038, AB252763, DQ647524. 